\title{
Las nuevas rutas de la energía de Rusia y la geopolítica mundial
}

Ana Teresa Gutiérrez del Cid *

\section{Resumen}

A momento de la desintegración, Rusia, la principal heredeAra de la URSS y principal productora de petróleo, comenzó su vida independiente con un enorme rezago económico y con la implantación de unas drásticas reformas económicas con el objetivo de que la economía transitara al capitalismo, en la cual el sector energético era un eje clave para la transformación. Vladimir Putin, el presidente de Rusia, encabezó la reestructuración y crecimiento de la industria petrolera del país hasta reposicionarla. A pesar de los esfuerzos de Estados Unidos y la Unión Europea por bloquear el desarrollo de las nuevas rutas energéticas de Rusia, el gobierno de Vladimir Putin ha logrado desarrollar este importante componente de su economía que le ha permitido junto con el desarrollo de un nuevo poderío armamentista reposicionarse como una gran potencia mundial.

Palabras clave

Geopolítica, Rusia, Petróleo y Gas, Rutas Energéticas.

Fecha de recepción: marzo de 2021
Fecha de aceptación: mayo de 2021

Profesora del Departamento de Política y Cultura. Área de Política Internacional. Universidad Autónoma Metropolitana-Xochimilco. Contacto: anateresagutierrezdelcid@gmail.com 


\title{
Russia’s New Energy Routes and World Politics
}

\author{
Keywords \\ Geopolitics, Russia, Oil and Gas, Energy Routes.
}

\begin{abstract}
:
At the time of disintegration, Russia - the main heir to the USSR and the principal oil producer- began its independent life with huge economic backwardness and with the implementation of drastic economic reforms to transition the economy to capitalism, in which the energy sector was a primary axis for the transformation. Vladimir Putin, the president of Russia, spearheaded the restructuring and growth of the country's oil industry until it repositioned. Despite the efforts of the United States and the European Union to block New Russian Energy Routes development, Vladimir Putin's government has been managing these economic and political strategies, by developing at the same time a new arms power, to position itself again as a great world power.
\end{abstract}

\section{Introducción}

Posterior a la desintegración de la Unión Soviética, Rusia experimentó un gran caos y descenso económico. Su industria petrolera se fragmentó debido a que existían fuentes de gas y petróleo en las repúblicas que se independizaron y, además, el robo de hidrocarburos era una constante, como resultado de la falta de un orden debido a la debilidad y corrupción del gobierno del primer presidente de la Rusia independiente, Boris Yeltsin.

El objetivo de este trabajo es analizar la trayectoria y las vías por las cuales, la Rusia actual superó el caos económico y político posterior a la desintegración soviética y reconstruyó el complejo energético, que tradicionalmente fue uno de los pilares de la Unión Soviética.

En efecto, en la actualidad Rusia ha reconstruido su industria petrolera y gasera, y la ha utilizado como instrumento de reposicionamiento para convertirse de nuevo en una gran potencia y para crear un entorno geopolítico favorable a sus intereses nacionales. En ese tenor, la pregunta de investigación es cómo ha logrado en tan pocos años volver a tener el estatus de gran potencia y lo que se intenta demostrar es que ha sido la industria petrolera y gasera la que le ha permitido volver a adquirir esa posición inter- 
nacional. Si bien ya no con la fuerza que tuvo la Unión Soviética, Rusia sí constituye hoy una de las grandes potencias mundiales.

La metodología de esta investigación consiste en analizar brevemente la historia de la industria petrolera de este país y después seguir el desarrollo de su reconstrucción en la era postsoviética del presidente Vladimir Putin para lograr analizar como este desarrollo ha logrado paulatinamente el ascenso de Rusia, no solo como potencia energética, sino también como potencia militar.

El actual desarrollo del complejo energético ruso ha superado al de la Unión Soviética, ya que ahora posee nuevos gasoductos como el Nord Stream 1 y el 2 aún en construcción; el gasoducto que va de Turquía a Europa y, finalmente, el gasoducto Fuerza Siberia que va de Rusia a abastecer China.

En este trabajo se tiene en cuenta el contexto geopolítico mundial en el que se desarrolla este avance, ya que se ha tratado de obstaculizar el reposicionamiento ruso por su competidor de la Guerra Fría, Estados Unidos, que junto con la Unión Europea se resisten a compartir la hegemonía mundial conseguida después de la desintegración soviética y que en la actualidad se encuentra en transición hacia una hegemonía compartida con Rusia y China.

En primera instancia se describe de forma breve el surgimiento y desarrollo del complejo petrolero y gasero en la Unión Soviética. En un segundo apartado se analiza el caos y derrumbe de esta industria como resultado de la desintegración soviética. En el apartado tres se plantea cómo el ascenso de Vladimir Putin al poder en Rusia permitió con la estrategia de su administración reconstruir la industria energética del país. En el cuarto apartado se analiza cómo se fue acotando a la inversión extranjera en este sector estratégico de Rusia, seguido de un apartado más en el que se analiza la posición de la Unión Europea, principal consumidora del gas ruso, por lo menos por países integrantes de esta. El artículo concluye con una anotación acerca de cómo han surgido las nuevas rutas de la energía de Rusia, el Nord Stream 1 y el Nord Stream 2, aún en construcción, y el gasoducto hacia China, así como el que parte de Turquía para comprender cómo Rusia se ha reposicionado como una gran potencia energética, militar y, por lo tanto, geopolítica. 


\section{La industria de energéticos en Rusia}

La historia de la Unión de Repúblicas Socialistas Soviéticas (URSS) y de la Federación de Rusia están indisolublemente unidas a la historia de la industria petrolera y, más tarde, gasera de este país. Las reservas probadas de petróleo de Rusia no son tan grandes: en 2017, 80 mil millones de barriles o 6.3\% de las reservas mundiales (Index Mundi Country Facts, 2017). A modo de comparación: el principal país petrolero del mundo, Venezuela, posee casi 301 mil millones de barriles, es decir, el 25\% de las reservas mundiales. Pero en términos de producción diaria, Rusia exporta 10 millones de barriles, que es casi la cantidad que exporta Arabia Saudita, es decir, 10.8 millones de barriles diarios. Además, otro energético vital para la economía de este país es el gas natural. Las reservas probadas son de 47.8 trillones de metros cúbicos en 2017 (Index Mundi Country Facts, 2017).

Antes de la desintegración soviética, el Complejo Energético lo constituían 2,400 empresas. Casi la mitad de estas se dedicaban a la producción de energía eléctrica y las restantes a la producción de carbón, petróleo y gas natural. Fue en la década de los setenta cuando el centro de producción de petróleo se trasladó a Siberia occidental, pero las principales áreas de consumo se encontraban en la parte europea del país. Los costos de producción aumentaban constantemente, porque se tenía que trabajar en condiciones extremas y el petróleo se transportaba a largas distancias.

Cuando dominaron Siberia occidental los petroleros fueron los primeros en llegar allí. Solo entonces inició la construcción de viviendas y otros servicios auxiliares. Esto significa que, además de la producción de petróleo, los jefes de los departamentos de producción de petróleo y gas en la región de Tyumen debieron construir establos, viviendas, escuelas y cines para garantizar condiciones de vida aceptables para los trabajadores y reducir la rotación de personal.

Durante la perestroika, la crisis económica ya existente en el sector desde mediados de los setenta se fue agravando, a pesar de las medidas instrumentadas para corregirla y que, paradó- 
jicamente, contribuyeron a profundizarla. En este contexto el petróleo fue utilizado para paliar los devastadores efectos de la economía, lo que a su vez incidió en el estancamiento del desarrollo del propio complejo energético soviético. Al momento de la desintegración, Rusia, la principal heredera de la URSS y principal productora de petróleo, comenzó su vida independiente con un enorme rezago económico y con la implantación de unas drásticas reformas económicas con el objetivo de que la economía transitara al capitalismo, en la cual el sector energético era un eje clave para la transformación.

Los problemas aumentaron debido a la inestabilidad política y la crisis étnica. Además, con el colapso de la URSS, se rompieron los lazos económicos con las empresas de las antiguas repúblicas que producían los derivados más importantes para la industria. Por ejemplo, Azerbaiyán producía el $40 \%$ de los equipos para campos petrolíferos; Ucrania se especializó en tuberías de petróleo; la flota de petroleros permaneció en los Estados bálticos, y Rusia tiene solo cuatro puertos (Novorossiysk, Tuapse, Nakhodka y Vladivostok) que exportan 40 millones de toneladas de petróleo por año.

Se señala esta dramática situación para evaluar los cambios ocurridos en el sector y en el país desde el año 2000, en el cual Rusia inició un significativo reposicionamiento económico, militar y de producción de petróleo y gas, debido a un cambio de estrategia política que le ha permitido un gran avance hasta reposicionarse como una gran potencia, sobre todo en los rubros energético y militar.

\section{La drástica crisis del sector energético después de la desintegración de la URSS}

En 1990 el Complejo Energético Soviético lo constituían, como ya se anotó, 2,400 empresas, que representaban el 5\% del total de las industrias existentes en la URSS. Este era un número reducido de empresas con altas escalas de producción, que disponían de una gran dotación de recursos. 
Entre las décadas de 1972 a 1992 el ritmo del crecimiento del Complejo Energético fue cada vez menor y las tasas medias de crecimiento fueron sistemáticamente inferiores a las del conjunto del sector industrial. La producción de combustibles fue importante hasta la mitad de los setenta, reduciéndose a partir de esta fecha hasta 50\% en el periodo de 1976 a 1980. Esta situación llevó al estancamiento del sector petrolero a mediados de los años ochenta, debido a los cada vez mayores problemas para la extracción de carbón y petróleo, lo que resultó en tasas negativas de crecimiento al final de esta década. Asimismo, la productividad del trabajo en el complejo energético era inferior a la del sector industrial y la desaceleración del crecimiento fue más intensa en las ramas energéticas que en el promedio del sector industrial.

En lo que respecta a las inversiones, el complejo energético había canalizado un enorme flujo de recursos. De 1970 a 1980 las inversiones se fueron incrementando a una media del 540\%, mostrando así un ritmo bastante más alto que el de las inversiones totales en la economía y el sector industrial (Palazuelos, 1993).

Entre 1986 y 1990 el monto de las inversiones realizadas en el complejo energético fue cuatro veces mayor al correspondiente a 1966 y 1970, y casi tres veces más que el de 1971-75 (Comité Estatal de Estadísticas, GOSKOMSTAT, 1991).

Una de las razones fundamentales de este aumento fue incrementar los ingresos en divisas a través de la exportación de combustibles a los países occidentales, creciendo la producción. La estrategia en el sector consistía en garantizar el nivel interno de abastecimiento y captar divisas por concepto de venta de petróleo y gas en el exterior. Estas ventas en el mercado mundial aportaban hasta dos terceras partes de las divisas que obtenía la Unión Soviética en los últimos decenios.

Con la privatización en noviembre de 1992, el gran sector energético en Rusia integró de manera vertical a las compañías petroleras. El sector del gas permaneció intacto bajo el monopolio de una gran compañía estatal denominada Gazprom. La producción de petróleo cayó en 1995 a 6.2 millones de barriles diarios, después de que en 1987 era de 12.5 millones de barriles (Telguian, 1999). 
Después de 1966, se construyeron 7 nuevas plantas. En la República Soviética Rusa se abrió la refinería de petróleo de Achinsk y las seis restantes en el territorio de las repúblicas de la Unión. Por lo tanto, después del colapso de la URSS, Rusia heredó las empresas más antiguas con una baja capacidad de procesamiento: alrededor del $67 \%$ frente al 85 y $95 \%$ en los países desarrollados. Se exportaba el crudo y era transformado en los países importadores (Pusenkova, 2012).

Con el objetivo de sostener y aumentar la producción petrolera con respecto a los anteriores niveles, fueron necesarias grandes cantidades de capital para desarrollar nuevos campos y extender la vida de los campos petroleros existentes con reservas bajas o agotadas. El fuerte crecimiento en los precios durante 1999 y 2000 dio a las compañías rusas un respiro en ganancias y muchas, incluso, han empezado a renovar su infraestructura y llevar a cabo nuevas perforaciones exploratorias. En adición, para desarrollar más la región de Siberia occidental, donde se extrae en la actualidad la mayoría del petróleo ruso, los productores están explorando el sector ruso del Mar Caspio (Sergeiev, 1998).

Debido a la carencia general de combustible líquido, ya desde 1992 comenzó una apropiación ilegal de petróleo y derivados directamente de los oleoductos por medio de perforaciones. Sobre todo, estaba generalizada la obtención de diésel del ducto que une a Bakú con el puerto de Batum en el mar Negro. De acuerdo con la información periodística, en este ducto había alrededor de mil fugas por perforación, por lo que se robaba una cantidad aproximada de $60 \mathrm{mil}$ toneladas de diésel al año (Sergeiev, 1998). También por esta causa dejó de funcionar el ducto de gasolina entre las ciudades de Grozny y Budenovsk. Además, este fenómeno también empezó a darse con el oleoducto macro que atraviesa el territorio de Bielorrusia.

Los grandes especuladores que se llevaban la gasolina en autocisternas por los caminos rurales a Estonia o por el río Dunai en caravanas con combustible a Siberia debido al embargo de la Organización de las Naciones Unidas (ONU), siguieron con su negocio. De Ucrania se llevaban a otros países más del 30\% del combustible líquido que Rusia le abastecía. 
Los primeros brotes de las relaciones de mercado en la industria petrolera nacieron incluso antes del colapso de la Unión Soviética, aunque las principales transformaciones del mercado en el sector ya comenzaron en la Federación de Rusia. El $1^{\circ}$ de enero de 1988, se introdujo la "Ley sobre las Empresas Estatales", que fue promulgada en la URSS con fecha 30 de julio de 1987, No 7284-XI, que otorgaba independencia económica a las empresas y que se puso en práctica después de la desintegración soviética (Pusenkova, 2012).

\section{La era de Vladimir Putin}

En 1999 Vladimir Putin se convirtió en primer ministro. Heredó un legado difícil: los oligarcas se sentían omnipotentes, un gobierno débil, la omnipresente corrupción y la burocracia. Pero, al mismo tiempo, el nuevo primer ministro tuvo mucha suerte. Los precios mundiales del petróleo subieron y la producción de petróleo comenzó a crecer.

El fuerte crecimiento en los precios durante 1999 y 2000 dio a las compañías rusas un respiro en ganancias y muchas empezaron a renovar su infraestructura y a llevar a cabo nuevas perforaciones exploratorias. Aunado a esto, para desarrollar más la región de Siberia occidental, donde se extrae actualmente la mayoría del petróleo ruso, los productores empezaron a explorar el sector ruso del Mar Caspio e intentaban hacer equipo con compañías extranjeras.

El futuro nivel de producción petrolera de Rusia sería definido por la habilidad de las compañías petroleras para desarrollar estos nuevos yacimientos en el Caspio, el Ártico y el Lejano Oriente ruso. Por otra parte, la carrera de Vladimir Putin despegó tan rápidamente que no tenía equipo propio para las elecciones del año 2000. El futuro presidente ruso comenzó a buscar conocidos, principalmente colegas de los servicios de seguridad y de los órganos del alcalde de San Petersburgo, en puestos clave, aunque al principio sus capacidades estaban limitadas por los hombres del gobierno de Yeltsin. Putin nombró a Semyon Vainshtok como jefe de la compañía petrolera Transneft. La compañía de Alexey 
Miller, que dirigió la construcción del Sistema de Tuberías Bálticas, inició la construcción de nuevos ductos, y Nikolay Tokarev, que venía del sector de la inteligencia rusa para el sector extranjero, llegó al puesto de vicepresidente de Transneft.

Después de que Putin fuera elegido presidente, sus protegidos aumentaron. Tokarev asumió el cargo de CEO de Zarubezhneft, otra compañía petrolera. Poco después de ser viceministro de energía, Miller se convirtió en presidente de la junta directiva de Gazprom, y Dmitry Medvedev e Igor Sechin, colegas de Putin en San Petersburgo, se convirtieron en jefes adjuntos de la administración presidencial.

En el primer mandato de la presidencia de Putin (2000-2004), la política energética aún no había tomado forma. Se aplicaron medidas para liberalizar el complejo de combustible y energía; continuó la privatización de las industrias del petróleo y el carbón, y se adoptó un programa de reformas de mercado en la industria de la energía eléctrica. Pero, al mismo tiempo, el Estado aumentó su influencia en el sector de petróleo y gas. Prohibió los oleoductos privados, fortaleció el control estatal sobre Gazprom y pospuso la reforma del monopolio del gas.

$\mathrm{Al}$ principio, el presidente y su administración no interfirieron particularmente en las políticas económicas y energéticas aplicadas por el primer ministro Mikhail Kasyanov. Su influencia creció, pero también los desacuerdos con el presidente aumentaron, incluyendo muchos aspectos sobre el complejo de combustible y energía.

Putin empezó a forjar un proyecto estatal de energía y Kasyanov era un político con aspiraciones y una visión privatizadora a favor del capital extranjero. Por este motivo no estuvieron de acuerdo sobre el papel del capital estatal y privado en la industria. Además, a principios de la década del 2000 las posiciones de cabildeo de las empresas privadas seguían siendo fuertes, lo que impulsó con éxito las iniciativas legislativas que les eran favorables. En la Duma estatal de la tercera convocatoria, el lobby de petróleo y gas incluyó a Vladimir Dubov, Sergey Kirienko, Boris Nemtsov, Dmitry Savelyev, Rem Khramov, Alexander Ryazanov, 
Victor Chernomyrdin, Valery Yazev y a Vladimir Medvedev, todos a favor de la privatización del complejo energético y de la intervención del capital extranjero en este.

Sin embargo, el proyecto de Putin planteó nuevas prioridades casi de inmediato. Por ejemplo, el poder político central comenzó a pesar sobre la independencia de las regiones. Para su puesta en práctica, se reformó el Consejo de la Federación, se crearon distritos federales, la aceptación de este nueva división administrativa-territorial resultó ser una condición para la victoria en las elecciones para gobernador, ya que el país estaba en peligro de desintegrarse debido a los conflictos en el Cáucaso y en otras regiones.

Las nuevas prioridades afectaron a las regiones petroleras. En 2001 Tatarstán perdió algunos privilegios otorgados a la república en 1994 y Tatneft, empresa de esta república que disfrutaba de importantes beneficios fiscales, comenzó a tener las mismas condiciones que todas las demás empresas.

En el segundo mandato de la presidencia de Putin, la actitud de las autoridades hacia la industria petrolera cambió drásticamente. Después de la renuncia de Kasyanov como primer ministro por las divergencias con Putin, fue nombrado Mikhail Fradkov, que ya no tuvo posibilidad de tomar decisiones clave sobre energía.

El Estado comenzó a intervenir activamente en la industria petrolera y la Duma estatal de la cuarta convocatoria, elegida a fines de 2003, era fundamentalmente diferente de sus predecesoras. La mayoría de los diputados estaba a favor del presidente Putin y permitió que cualquier ley se aprobara rápidamente y sin problemas. Así, aunque había suficientes representantes petroleros en esta Duma, preferían seguir la línea general de poder y actuaban lo más silenciosamente posible si no coincidían con las directrices de la nueva estrategia energética.

De esta forma, el gas y el petróleo empezaron a formar parte de la seguridad nacional y de la política exterior de la Federación de Rusia. Se elaboró el documento "Estrategia Energética Rusa para el periodo 2005 hasta el año 2020", en el que se argumenta que "la seguridad energética es el elemento más importante de la seguridad nacional de Rusia” (Gobierno de Rusia, 2005), por lo 
que el Estado debe tener un papel activo en el sector energético para proteger a Rusia de las amenazas internas y externas.

En el documento "Revisión de la Política Exterior de la Federación Rusa", elaborado por el Ministerio de Relaciones Exteriores de Rusia en 2007 se plantea que "El principal logro de los últimos años es la recién adquirida independencia de la política exterior de Rusia. Ha surgido la necesidad de comprender la nueva situación, incluso a nivel doctrinal" (Intelectualnaia Rossia, 2007).

Además, se argumenta que:

En el trabajo de política exterior en las nuevas condiciones, es más importante que nunca, partir de las necesidades urgentes del país, de los intereses de los ciudadanos rusos, tener en cuenta los factores reales que conforman la seguridad y la prosperidad nacional de Rusia. Para esto, es necesario aprovechar plenamente las ventajas competitivas de nuestro país, que son muchas [...] Al mismo tiempo, varios países que se unieron a la Unión Europea en 2004 están intentando "aprovechar" la membresía para realizar sus tareas políticas en contra de Rusia, convirtiendo las relaciones Rusia-UE en un "rehén" de sus propios intereses nacionales (Intelectualnaia Rossia, 2007).

Por otra parte, se divide el oficio diplomático en cuatro tipos de diplomacia: multilateral, económica, humanitaria y de cooperación. La diplomacia económica se define como un instrumento para asegurar la integración de Rusia en los mercados globales y el uso de los recursos energéticos como instrumento de política exterior para afianzar el interés nacional.

En otro documento: "Estrategia 2020" publicado en 2008, se sostiene que "la energía es un aspecto vital de la seguridad nacional, como instrumento de poder y como posible amenaza en el caso de que los actores sin recursos intenten arrebatar a Rusia los suyos" (Milosevich-Juaristi, 2016)

La influencia política de los oligarcas y los gobernadores de la Federación, cuyo poder se basaba en los ingresos del petróleo, comenzó a ser limitada. En un esfuerzo por privar del petróleo 
a los "barones" regionales y, por lo tanto, de su base económica en agosto de 2004, la Duma del Estado adoptó enmiendas a la Ley "Sobre el subsuelo", que abolió las iniciativas locales en el uso del subsuelo y de la extracción de energéticos. El poder central adquirió el poder absoluto en el campo de las licencias: ahora la decisión de emitir una licencia debía ser tomada por la comisión federal, a la que se invitó a representantes de la Federación.

\section{Empresas extranjeras}

Debido al aumento de los precios del petróleo y al fortalecimiento de las compañías petroleras rusas, la actitud de las autoridades hacia los actores extranjeros en la industria petrolera también cambió desde el ascenso del gobierno de Putin al poder: se les asignó el papel de socios minoritarios y los inversionistas extranjeros trabajaban en Rusia en casi cualquier condición aceptable.

Una fuerte señal de la nueva actitud hacia la participación de los extranjeros en el desarrollo del subsuelo ruso, fueron los eventos en torno al bloque denominado Kirinsky de depósitos del proyecto Sakhalin-3 en la isla Sahalin. Los bloques Sakhalin-3 se pusieron a concurso en 1993. El bloque Kirinsky fue concesionado a Mobily Texaco y el East-Odoptinsky y el Ayashsky a Exxon.

En 1997 Mobily Texaco se vieron obligados a ceder un tercio de su proyecto a las compañías rusas Rosneft y Sakhalinmorneftegaz. Y en enero de 2004, el gobierno privó a Exxon, Mobil y a Chevron del derecho a trabajar en el bloque Kirinsky, motivado esto por el hecho de que los inversionistas no tenían un documento específico de concesión a esta sección del campo petrolero.

Tanto el Estado como las compañías rusas dejaron de autorizar el papel principal que los extranjeros desempeñaban en los grandes proyectos de petróleo y gas, especialmente en el Lejano Oriente, estratégicamente importante. Aunque en esta región solo se implementaron con éxito los proyectos Sakhalin-1 y Sakhalin-2, que fueron gestionados por empresas internacionales.

El "patriotismo del petróleo y el gas" se manifestó claramente en el concepto de los depósitos estratégicos. En 2005, se decidió 
actualizar la anterior Ley del subsuelo. Una de las enmiendas más importantes fue limitar el papel de los extranjeros en el desarrollo de depósitos estratégicos: en lo sucesivo, solo las empresas rusas podían ingresar, en cuyo capital la participación del capital extranjero debía ser inferior al 50\% y en los consejos de administración, los extranjeros debían ser menos de la mitad.

La estrategia de Putin para el renacimiento económico consistió en volver a estatizar las empresas públicas pertenecientes a la era de la Unión Soviética y que después fueron privatizadas durante la era Yeltsin, sobre todo, las industrias del complejo militar industrial y las de energéticos. Así, ha recuperado sistemáticamente el control de las empresas que explotan el petróleo y el gas en su territorio, y cerca del 80\% de las reservas mundiales de petróleo y gas en Rusia les pertenecen hoy a compañías de propiedad estatal (Tsujlo, 2005).

Con esta situación, la siguiente estrategia del gobierno ruso ha sido utilizar sus recursos energéticos como instrumento de reposicionamiento geopolítico. En este marco, la compañía estatal de gas Gazprom, primer exportador de gas natural del planeta, tiene como objetivo controlar el tránsito del gas hacia Europa Central y Occidental, Asia y Medio Oriente.

El criterio estratégico sobre la importancia de los campos petroleros y gaseros se ha ajustado constantemente bajo la presión del Servicio Federal de Seguridad. Para el petróleo el umbral se redujo de 150 millones a 70 millones de toneladas; para el gas de 1 billón a 50 mil millones de metros cúbicos para explotación extranjera. La disminución en las concesiones también fue promovida por Gazprom y Rosneft, que en compañías extranjeras solo vieron socios técnicos y financieros.

Las tendencias particularmente vívidas de nacionalización y las restricciones sobre el papel de los actores extranjeros en la industria petrolera se manifestaron en la política de desarrollo de nuevas provincias de exploración de petróleo y gas: Siberia oriental, Lejano Oriente y la plataforma continental.

A futuro el gas sustituirá al petróleo en la mayor parte de las exportaciones rusas, ya que Rusia posee una tercera parte de las reservas mundiales de gas y a través de la compañía estatal $G a$ - 
zprom, Rusia ya es la principal exportadora mundial de gas y es la principal proveedora de la Unión Europea.

Debido a esto, el gobierno ruso posee hoy un superávit presupuestario después del crónico déficit de los noventa. Este es uno de los grandes logros del gobierno de Vladimir Putin desde que llegó al poder en el año 2000. Y se debe fundamentalmente a que se ha asegurado que la mayor parte de las entradas por concepto de la venta de energéticos ingrese a las arcas del Estado para recuperar la industria, y no a las manos de los oligarcas que, en los noventa, después de la desintegración de la URSS se quedaban con las ganancias, lo que les permitió acumular grandes fortunas, además de evadir impuestos. Por otra parte, por las transferencias de precios pudieron amasar grandes fortunas, mediante la creación de compañías off-shore para comprar petróleo a bajo precio en los lugares de producción y luego revenderlo a través de intermediarios.

\section{La Unión Europea y las rutas de energía de Rusia}

En 2009, después de que Rusia cerró la llave del gas a Ucrania, debido a la falta de pago de este país por el consumo de gas natural, la Comisión Europea propuso tomar nuevas directrices. Así, en septiembre de 2009 fue tomada la decisión de aprobar un tercer paquete energético. Su objetivo era el aumento de la competencia en el mercado de gas por medio de la aparición de nuevos actores y para lograr también la disminución del costo de los energéticos. Para estos fines se propuso a las compañías de gas y petróleo vender las redes de gasoductos a operadores diferentes de los productores. La actual actividad de Gazprom coincide en su totalidad con esta forma de trabajo, porque el gasoducto denominado Turquish Stream, el cual se analizará más abajo, solo llega a la frontera turco-griega.

En el marco del Tercer Paquete Energético, la construcción de nuevos proyectos de transporte de gas se reglamenta en el Artículo 13.2 de esta legislación, que establece que cada operador de gasoductos está obligado a construir suficientes capacidades 
transfronterizas para unir la infraestructura europea de gasoductos económicamente y en su funcionamiento. Sin embargo, los procedimientos para la realización de este formato hasta la fecha no están definidos legalmente y ahora se elaboran en los marcos de la preparación de correcciones al Código de Redes del mecanismo de distribución de las capacidades de los gasoductos magistrales. Por lo que todas las inversiones en la construcción de nuevos gasoductos en 2018 deben contemplar esta norma, pero a la vez existe un vacío legal.

El 25 de febrero de 2015 la Comisión Europea aprobó una estrategia de creación en Europa antes del 2020 de un mercado energético unido. La iniciativa privada debe crearlo con base en la idea fundamental de Europa de adquisición de gas por medio del mercado. Sin embargo, este tipo de contratos Spot, por ejemplo de gas de Catar, no son constantes, ya que el precio del gas puede ser más barato, pero también más caro. El gas ruso es más barato bajo contratos de largo plazo, ya que su precio se define por el precio del petróleo y existe la posibilidad de acumular reservas en sus propios contenedores europeos.

Hay que señalar que, en la estrategia de creación de un mercado energético único antes de 2020, existía el objetivo de ampliar la participación de la energía alternativa. En parte se esperaba que esta participación aumentara del actual $14 \%$ al 20\%. Sin embargo, la energía alternativa se basa fundamentalmente en las dotaciones del presupuesto gubernamental, por eso, en condiciones de estancamiento de la economía europea es preferible usar el gas derivado del petróleo.

Según Milosevich-Juaristi:

Desde los años 60 hasta 2009, los países de la UE no consideraron que la dependencia del gas ruso fuera una amenaza geopolítica. Sin embargo, cuando las disputas por los precios del gas entre Rusia y Ucrania condujeron a la interrupción de los suministros rusos a Europa a través de Ucrania en enero de 2009, se demostró que la seguridad energética europea está vinculada a los objetivos de la política exterior rusa y a los intereses de las 
compañías, dirigidas según la UE de manera poco transparente y controladas por los Estados: Gazprom y Rosneft por Rusia y RosUkrEnergo por Ucrania. Rusia mostró astucia al usar el control directo del gas y de las redes que lo distribuyen como instrumento de influencia y presión política (Milosevich-Juaristi, 2019).

Por lo que vemos, como la actitud de Ucrania de no pagar por el gas y el entramado geopolítico europeo y estadounidense que intentan que Ucrania ingrese a la Organización del Tratado del Atlántico Norte (OTAN), impiden que las nuevas rutas rusas se desarrollen normalmente.

\section{El nuevo gasoducto Nord Stream 2}

Como se ha anotado, una de las razones del poderío ruso es su gran industria petrolera y gasera y tiene una importancia clave en el reposicionamiento político y económico del país después de la desintegración soviética. El principal gasoducto histórico de Rusia es el gasoducto Drushba por el cual ha exportado tradicionalmente gas a Europa.

Sin embargo, en el 2006 Ucrania, antigua república federada soviética, dejó de pagar por el gas y planteó su objetivo de ingresar a la OTAN. Rusia le cortó el suministro de gas en pleno invierno y afectó mucho a Europa en este contexto geopolítico ya mencionado. Por este motivo, el principal socio económico ruso en la Unión Europea, Alemania, decidió conjuntamente con Rusia crear un nuevo gasoducto que se llamara Nord Stream 2. En 2011 entró en funcionamiento el gasoducto Nord Stream I, que va de un puerto ruso a un puerto alemán. Este proyecto inició después del segundo conflicto de Rusia con Ucrania en el invierno de 2009, cuando esta intentó nuevamente ingresar a la OTAN.

El proyecto ruso-alemán denominado Gasoducto del Norte o Nord Stream, une el litoral ruso en el Mar Báltico en el puerto de Viborg con el litoral báltico alemán en el puerto de Greisfwald y transita por el lecho del Mar Báltico. Este gasoducto le permitió a Rusia evadir el tránsito tradicional por Ucrania, Bielorrusia y 
Polonia. Además, une las redes de gas de Alemania y otros países de la UE con la red abastecedora de Rusia. Los principales mercados de abastecimiento son Alemania, Reino Unido, Países Bajos, Francia y Dinamarca.

El campo Ninetz en la isla rusa de Yamal es el que abastece este gasoducto. En su construcción trabajaron Gazprom y, por parte de Alemania, las compañías BASF y EON. También se extraen recursos del yacimiento Shtokmanovsk en el Mar de Barentz, con las compañías Gazprom, la francesa Total y la noruega Statoil Guidro. Las reservas de gas de este yacimiento contabilizan cerca de 3.7 trillones de metros cúbicos.

La longitud del gasoducto es de $1,200 \mathrm{~km}$. El costo de la obra fue aproximadamente de 10,000 millones de dólares. Se estima que por el gasoducto transitan 55 mil millones de metros cúbicos anualmente. Gazprom participó con el 51\% del capital y BASF y EON tuvieron una participación de 20\% y, además, participó una compañía holandesa y otra francesa.

Sin embargo, frente a la construcción del Nord Stream, Europa se dividió en países que sí querían la construcción y los que deseaban detenerla. Los países a favor de la construcción fueron, en primer lugar, Alemania, que se abastece en un $40 \%$ del gas ruso, y también Francia, Países Bajos e Italia. Los países que se resistían a su construcción fueron: Polonia, Letonia, Lituania, Estonia y Suecia, pero el principal opositor fue Estados Unidos que retóricamente afirmaba que era un asunto europeo.

Su verdadera posición desde que surgió el proyecto en 2006 fue argumentada por el presidente George W. Bush, quien afirmó que la construcción de este ducto afectaba la seguridad energética europea, por hacer más dependiente a Europa del gas ruso. Bush declaró también que su oposición se debía a que la seguridad energética europea constituía un interés estratégico de Estados Unidos.

Así, a inicios de 2007 algunos senadores estadounidenses influyentes, incluido el ahora presidente Joseph Biden, llevaron al Congreso de Estados Unidos un proyecto de ley denominado "Sobre la diplomacia energética y la seguridad". Este proyecto tenía como uno de sus principales planteamientos el "apoyo de las 
aspiraciones de las jóvenes democracias como Ucrania y Georgia hacia la independencia energética" (Khasanov, 2017).

En el texto se apuntaba que una prioridad para estos gobiernos era la independencia energética con respecto a Rusia y este objetivo jugaba un papel clave en las relaciones de Estados Unidos con estos países; por lo que la posición estadounidense consistía en que este proyecto no debería ser realizado, porque era predominantemente un proyecto ruso que le daba a Moscú beneficios económicos y políticos sustanciales y, sobre todo, fortalecería las posiciones rusas en Europa, la unirían más a Rusia y resultaría en que los importadores europeos que cada vez demandarán más gas se volverán totalmente dependientes del gas ruso; como resultado, Rusia podría aún más utilizar los energéticos como un medio de "chantaje político".

Para la óptica estadounidense, además, no solo la independencia de Europa occidental peligra, sino también la de Europa oriental, ya que al evadir el territorio de Polonia, Ucrania y los Bálticos hará que Rusia tenga más libertad de negociación con estos países, pues ya no tendrá la preocupación de que son tránsito del gas ruso y debe negociar con estos. Para evitar la construcción de este gasoducto, Estados Unidos amenazó a Alemania con represalias si persistía en construir el proyecto.

El Ministerio de Relaciones de Rusia se quejó de esta falta de diplomacia en el trato a Europa y a la misma Rusia y la respuesta del Departamento de Estado fue que Estados Unidos lograría la diversificación de abastecimiento de gas a Europa para disminuir su dependencia de Rusia (Financial Times, 2006).

Después de estas declaraciones tuvo lugar un activo lobby en Estados Unidos para construir el proyecto denominado BTC - gasoducto que parte de Bakú, Azerbayán y transita por Tbilisi, capital de Georgia y llega al puerto turco de Ceyhán- que evade el territorio ruso, disminuyendo así la influencia de Rusia en el Mar Caspio.

A este respecto, el politólogo alemán, Alexander Rar, considera que la politización de la construcción del gasoducto Nord Stream era innecesaria, ya que los ductos existentes no eran suficientes para garantizar el abastecimiento de gas de la UE (Rar, 2011). 
A pesar de la férrea oposición de Estados Unidos, el gasoducto Nord Stream 1 fue construido en 2011, lo que le ha permitido a Rusia exportar gas sin problemas con países de tránsito y a Alemania un abastecimiento seguro y accesible. De esta manera, el proyecto ha sido favorable a ambas naciones, al grado de que en 2015 en el Foro Económico de San Petersburgo, surgió la propuesta de construir otro gasoducto con el nombre de Nord Stream 2. Este nuevo proyecto prevé la construcción de un gasoducto paralelo al primero con una extensión de 1,200 kilómetros y una capacidad de 55 mil millones de metros cúbicos anuales con el mismo objetivo de exportar el gas natural ruso a Europa.

La construcción iría también a través de aguas territoriales de cinco países: Rusia, Finlandia, Suecia, Dinamarca y Alemania, y debía iniciar su construcción en 2018 y se pondría en explotación en diciembre de 2019 e inicios de 2020. El gasoducto ya está construido, pero Estados Unidos y la UE han prohibido su puesta en funcionamiento. El argumento de Rusia es que se trata de un proyecto puramente económico, tratando de evitar nuevamente la politización, lo cual, desde luego, es un argumento retórico.

Al igual que con el Nord Stream 1, se creó una sociedad para la construcción de este ducto, en la cual Rusia posee el 51\% de las acciones y participan Alemania, Austria, Países Bajos y Francia. En diciembre de 2016 el Consejo directivo de Gazprom favoreció la creación de esta compañía accionaria. Gazprom participó con el 50\% y el 3 de febrero de 2017, el Consejo Directivo de Gazprom dio el visto bueno a la compra por 30 millones de euros al restante paquete de $50 \%$ de las acciones. El único accionista del Nord Stream 2 es Gazprom. Esto permitió crear la base para definir la futura cooperación con los países occidentales. El 24 de abril de 2017 se firmó un acuerdo con 5 compañías europeas: la alemana Wintershall, la austriaca $O M V$, la holandesa-británica Royal Dutch Shell y la francesa Engie para financiar el gasoducto por 4.75 mil millones de euros y acordaron pagar a futuro $9.5 \mathrm{mil}$ millones de euros.

A este proyecto también le surgieron varios opositores, descontentos con los términos económicos, políticos y legales. Estos países 
son: Ucrania, Polonia, los Países Bálticos y Estados Unidos. A este respecto, el Director del Ministerio de Energía de Ucrania, Igor Nasalik, declaró que esta no era una vía alternativa a Rusia para importar gas a Europa, y definió al proyecto como antiucraniano, totalmente político. El director de la compañía de gas de Ucrania, Naftogaz Ucraini, Yuri Vitrienko lo denominó "una arma política del Kremlin”.

En una entrevista con un medio alemán, Vitrenko argumentó que "las sanciones estadounidenses o la posición de Dinamarca pueden retrasar y complicar aún más la situación con el Nord Stream-2. Las sanciones estadounidenses son un factor importante. Un factor importante también es la posición del lado alemán, que ha evolucionado recientemente. Y nos parece que los políticos alemanes pueden darse cuenta de que este proyecto es realmente peligroso tanto para Alemania como para toda Europa" (Deutsche Welle, 2018).

En los años 2015 y 2016, Polonia, la República Checa, Eslovaquia, Hungría, Rumania, Croacia, Grecia y las Repúblicas Bálticas dos veces le enviaron al líder de la Comisión Europea, Jean Claude Junker, una carta con la exigencia de prohibir el proyecto por ser una amenaza a la seguridad energética de Europa y no compatible con la estrategia europea de desarrollo energético alternativo. Los opositores al proyecto también consideraron que este proyecto no era adecuado para el Tercer Paquete Energético de la UE y declararon que Gazprom era el arma económica de Rusia (Maskevich, 2018).

Varsovia consideró a su vez que se trataba de una amenaza política porque tenía la posibilidad de aumentar la influencia de Moscú y Berlín en el mercado de gas europeo. A este respecto el analista económico Igor Maskevich plantea que "El proyecto es claramente beneficioso para Alemania, que recibe una tubería directa y a bajo costo (décimas de un por ciento de su PIB) y así se convierte en el mayor centro de gas en Europa. Por lo tanto, Berlín está promoviendo poderosa y constantemente el proyecto ruso, ya que se ha asegurado el apoyo de sus aliados" (Maskevich, 2018). 
También Estados Unidos es de nuevo un fuerte opositor a este proyecto. El entonces vicepresidente Joseph Biden, declaró en 2016 que el Nord Stream era un mal trato para Europa y que esta ya podía comprar gas natural de esquisto producido por su país (RT, 2016). Además, se aprobó la ley CAATSA, ley contra los adversarios de Estados Unidos mediante sanciones. Es una ley federal de los Estados Unidos que impuso sanciones a Irán, Corea del Norte y Rusia. El proyecto de ley se aprobó el 27 de julio de 2017, 98-2 en el Senado, después de haber aprobado la Cámara el documento 419-3 (US Department of Treasury, 2017).

$\mathrm{Su}$ objetivo es supuestamente: "contrarrestar la agresión de Irán, Rusia y Corea del Norte a través de medidas punitivas" (Singotiya, 2018). En específico, la Ley apunta a imponer sanciones a los sectores rusos de petróleo y gas, defensa, seguridad y financieras. El gobierno estadounidense considera que Rusia invadió militarmente a Ucrania e intervino en las elecciones presidenciales estadounidenses de 2016 y de allí le aplica estas sanciones.

Así, se faculta al presidente de los Estados Unidos para imponer al menos 5 de las 12 sanciones enumeradas en la Ley a las personas involucradas en transacciones significativas con el sector ruso de defensa e inteligencia. El presidente de los Estados Unidos:

- Puede suspender la licencia de exportación relacionada con municiones, tecnología de doble uso, artículos relacionados con la energía nuclear.

- También prohíbe la inversión estadounidense en la entidad/persona sancionada.

- Prohibición de prestamos ofrecidos por instituciones financieras internacionales.

- Restricción de visa para la entidad sancionada.

- Suspende la asistencia ofrecida por los bancos estadounidenses.

A finales de octubre de 2017, el Ministerio de Finanzas de Estados Unidos emitió un comunicado en el cual prohibió a los sujetos físicos y jurídicos en su jurisdicción desde enero de 2018 a cooperar con compañías rusas en los proyectos energéticos. 
El 29 de enero de 2018, la administración de Donald J. Trump presentó al Congreso cinco informes según lo ordenado por CAATSA, incluidos los del programa de misiles de Irán, las personas sancionadas de Corea del Norte y dos versiones (clasificadas y no clasificadas) del informe con respecto a figuras políticas y oligarcas de alto rango en la Federación Rusa y sus entidades paraestatales.

Por esta ley el gobierno de Estados Unidos puede aplicar sanciones extraterritoriales a las compañías de terceros países, que colaboran con organizaciones rusas que están en una lista de compañías sancionadas. Esta ley se aplica a las compañías europeas que participan en los proyectos rusos del gas, el mantenimiento o la modernización de los gasoductos con el objetivo de exportar recursos energéticos desde Rusia (incluyendo la infraestructura de gas de Ucrania y del Mar Caspio). Debido a esta disposición, las inversiones de las compañías OMV de Austria y de la francesa Engie se vieron en peligro.

El 15 de marzo de 2018, 39 senadores estadounidenses pidieron al gobierno que utilizara todos los instrumentos que poseía, incluida la Ley CAATSA para bloquear la construcción del nuevo gasoducto, aduciendo que el Nord Stream 2 representaba un paso atrás en los intentos de diversificar el abastecimiento de gas y además perjudicaba a Ucrania. Los senadores y los funcionarios estadounidenses no reconocen que también están defendiendo los intereses de las compañías estadounidenses exportadoras de gas por medio del proyecto de gas natural licuado construido en Polonia.

Así, en una visita a Polonia del 6 de julio de 2017, previo a la Cumbre del G-20 en Hamburgo, Alemania, en una reunión denominada Iniciativa de los Tres Mares en Varsovia, Donald Trump les dijo a los líderes presentes que deberían tomar las exportaciones de energía de los Estados Unidos como una alternativa a la dependencia del gas ruso. Y el lema fue "más caro, pero más seguro".

La Iniciativa de los Tres Mares es una creación de 12 países de Europa Central y Oriental para coordinar políticas energéticas, entre otros objetivos. Trump le dijo a su audiencia polaca, claramente refiriéndose a Rusia: "Nos comprometemos a garantizar su acceso a 
fuentes alternativas de energía, por lo que Polonia y sus vecinos nunca más serán rehenes de un solo proveedor de energía” (Ansley, 2017).

La parada de Trump en Varsovia en ruta hacia la cumbre del G20 fue calculada para alimentar los sueños polacos de respaldo estadounidense para bloquear el gasoducto ruso-alemán Nord Stream 2 en el lecho del mar Báltico, desde Ust Luga al sur de San Petersburgo, hasta Greifswald, Alemania, a mitad de camino entre Berlín y Hamburgo, a 80 kilómetros de la frontera polaca.

En sus reuniones con el gobierno polaco, Trump habló sobre la infraestructura de gas natural licuado (GNL) y las enormes posibilidades de importarlo de su superávit de gas de esquisto. Por su parte Polonia perdería la tarifa de tránsito del gasoducto que pasa por su territorio y atraviesa Ucrania si se concreta el funcionamiento del Nord Stream 2. Pero el gas de esquisto de Estados Unidos enviado por tanques especiales desde un número muy limitado de terminales de GNL existentes en la Costa Este y el Golfo de México, no es barato.

En junio de 2017, el primer embarque estadounidense de GNL llegó a Polonia desde la planta Saiten Pass de Cheniere Energy en Louisiana. Y no fue barato. Los consultores de energía estiman que el precio en la terminal polaca de GNL en Swinoujscie es de \$5.97 por millón de unidades térmicas británicas (BTU). El mismo gas en el mercado estadounidense hoy cuesta alrededor de $\$ 3$ por millón de BTU. Se estima que el gas ruso a Alemania cuesta alrededor de $\$ 5$ por MBTU.

Enviar gas en tanques de GNL es un proceso costoso. Requiere la construcción de terminales especiales de GNL tanto en el puerto de origen como en el de destino. El gas debe primero transformarse en un estado líquido frío a aproximadamente $-260^{\circ} \mathrm{F}$, y cargarse en los buques tanque especialmente fabricados. En el destino, se requiere una terminal de GNL especial, similar a la de envío, donde el gas puede cambiarse nuevamente de estado líquido a gas para el consumo final. Todo esto es bastante costoso en comparación con las rutas de gasoductos rusos y europeos.

Polonia intenta así reemplazar a Ucrania como el país tránsito de gas a la UE, con gas de Noruega y gas de GNL de Estados Unidos 
y tal vez con gas de Catar si Washington no logra interrumpirlo mediante sanciones sauditas. Así, Polonia está construyendo una estrategia para convertirla en el nuevo centro de concentración y distribución de energía de Europa central para reemplazar el gas ruso. Este es el corazón del proyecto Three Seas Initiative. La nueva terminal de GNL que fue construida a un costo de mil millones de dólares puede aceptar 5 mil millones de metros cúbicos de gas por año, alrededor de un tercio del consumo anual de gas de Polonia e intenta aumentar esta cantidad. La estrategia exige hacer de Polonia un centro de gas natural para Europa Central al unir Polonia con Lituania, Ucrania, Eslovaquia y la República Checa a través de interconectores.

Los gobiernos de Alemania y Austria inmediatamente registraron una vehemente oposición a las últimas sanciones estadounidenses por razones obvias. El 15 de junio, los ministros de asuntos exteriores de Alemania y Austria emitieron una declaración conjunta inusualmente crítica de los Estados Unidos. Declararon en términos muy fuertes que el suministro de energía de Europa es un asunto de Europa, no de los Estados Unidos de América. No podemos aceptar la amenaza de sanciones extraterritoriales ilegales contra las empresas europeas que participan en el desarrollo del suministro energético europeo [...] Austria boicoteó la aparición de Trump el 6 de julio ante la Iniciativa Three Seas para señalar su desaprobación por las conversaciones sobre gas de Estados Unidos (Carbajosa, 2017).

Las acciones estadounidenses en Siria y sus negociaciones con Arabia Saudita contra Irán y Catar son parte de esta estrategia. La eliminación o la fuerte reducción de las exportaciones de GNL de este país, debido a que está bloqueado porque empezó a negociar la explotación de un campo de gas con Irán y fue también sancionado, han afectado otros posibles abastecimientos de gas para la UE.

En realidad, el bloqueo de Catar por parte de los saudíes no tiene como objetivo detener a los terroristas radicales. Su objetivo es mantener el gas iraní, catarí y, posiblemente, sirio, fuera del mercado de gas de la UE, que se estima se convertirá en el mayor consumidor mundial de gas en los próximos años. Para Washing- 
ton, Polonia y su Iniciativa de los Tres Mares no son más que una jugada de ajedrez en un juego geopolítico más grande.

\section{La posición de la UE sobre el Nord Stream 2}

Según Isabel Gacho Carmona, del Centro Español de Estudios Estratégicos: Europa ha dependido de la energía rusa desde la Guerra Fría. Para llegar a esta situación, Moscú ha seguido una llamada "diplomacia del gasoducto", instrumentalizando sus recursos energéticos con fines geopolíticos y geoeconómicos abordando a los países europeos de manera bilateral. (Carmona, 2019, p. 3).

Según Carmona:

El hecho de que Alemania sea el Estado miembro tras el proyecto es especialmente preocupante. Si la locomotora de la Unión actúa unilateralmente, ¿qué nos queda? La respuesta de Alemania a la estrategia bilateral de Rusia está debilitando las posibilidades de una solución integral y común basada en la solidaridad entre los miembros. Este es solo un ejemplo más de un problema mayor de la UE, un problema que hemos visto en muchos ejemplos de intereses nacionales que socavan los enfoques comunes.

El hecho de que no hubiera una necesidad real para la construcción de este gasoducto muestra el triunfo del bilateralismo y la Realpolitiky, también muestra el profundo problema subyacente: la falta de solidaridad entre los miembros, en este caso, hacia los países de Europa Central y Oriental (Carmona, 2019, p. 16).

\section{El gasoducto Turkish Stream}

Turquía es uno de los mayores consumidores de gas ruso. En la actualidad, sus suministros a la república se llevan a cabo a través del gasoducto Blue Stream y el gasoducto Trans-Balcánico. La destrucción del avión de combate $\mathrm{Su}-24 \mathrm{M}$ ruso en Siria, incidente ocurrido el 24 de noviembre de 2015, consistió en que este bombardero de primera línea de la Fuerza Aérea Rusa, parte del grupo de la aviación rusa en Siria, fue derribado por un misil aire-aire lanzado por el caza F-16C de la Fuerza Aérea Turca en el área de la frontera si- 
rio-turca a una altitud de unos 6,000 metros, y cayó en el territorio de Siria, provincia de Latakia en la región de Baiyrbuja.

Ya en febrero de 2016, el presidente turco Recep Erdogan quería propiciar una incursión unilateral en Siria, para no quedar fuera de la ecuación que decidirá el futuro de ese país, en clara provocación a Rusia, pero la iniciativa fue rechazada por el Ejército turco, así como por Estados Unidos.

El Subsecretario de Relaciones Exteriores ruso, Alexéi Meshkov declaró como consecuencia de este suceso que: "en lugar de continuar negando su responsabilidad en el incidente y denigrando las acciones legítimas de Rusia en Siria, las autoridades turcas deberían hacer lo que dictan las normas del derecho internacional: pedir disculpas, pagar una indemnización y presentar garantías de que no se repetirá en el futuro" (Evseev, 2015, p. 44).

Meshkov consideró que "los hechos objetivos indican que fue una acción anti rusa autorizada”. El diplomático ruso aseguró que Ankara "ha violado el principio jurídico internacional de no utilización de la fuerza en las relaciones internacionales consagrado en la Carta de las Naciones Unidas y el Tratado de Relaciones Básicas de la Federación de Rusia y la República de Turquía desde el 25 de mayo 1992" (Evseev, 2015, p. 46).

Pero a finales de junio del mismo año, en un hecho inesperado, Erdogan, que sufrió un intento de golpe de Estado por parte de una oposición proclive a Estados Unidos, cambió su actitud hacia Rusia y se disculpó con Moscú, asegurando que el derribo del caza ruso no había sido intencional, una declaración que distó del cáustico tono que había caracterizado las declaraciones del mandatario turco sobre Rusia (Evseev, 2015).

A finales de noviembre de 2014, las relaciones de la UE con Rusia empeoraron drásticamente debido a la crisis con Ucrania y la reabsorción de Crimea por Rusia. Este acto de Rusia fue contrario al Memorándum de Budapest (1994), según el cual, Rusia, el Reino Unido y los EU se comprometían al respeto de las fronteras de Ucrania, pero ante el derrocamiento en 2014 del presidente constitucional del partido de las Regiones, Víctor Yanukovich y la franca intromisión de Alemania y EU en la denominada "revolución de 
Maidan" en esa fecha, el gobierno ruso consideró que el siguiente paso de estas potencias era expulsar a la Flota Rusa del Mar Negro de Crimea y establecer una base de la OTAN en su territorio, lo cual era impensable para el gobierno y los militares rusos.

Por lo que, desde el punto de vista geopolítico, para la estrategia estadounidense y la de la Unión Europea (UE), la separación de Ucrania con respecto a Rusia es una de sus prioridades y es una estrategia puesta en práctica que lleva 20 años. Esta estrategia ha consistido en impedir que Rusia tenga nuevamente influencia en Ucrania por medio de varias tácticas, desde el financiamiento de grupos de oposición, creación de redes sociales pro estadounidenses y la realización de la revolución naranja en 2004, cuando Víctor Yushenko, un presidente pro estadounidense ascendió al poder: Los sucesos de 2014 fueron la continuación de esta estrategia.

En el país reina una división entre la parte occidental y la oriental, debido a que uno de los primeros decretos del Parlamento ucraniano después del golpe de Estado, fue la supresión del idioma ruso como lengua también oficial a la par que el ucraniano, y los ultranacionalistas ucranianos llevaron a cabo una política de discriminación a la población étnicamente rusa de Ucrania, que vive en el sureste del país, denominada como Cuenca del Don, así como el quitarle sus derechos políticos. Debido a esto, en las principales ciudades del sureste de Ucrania: Járkov, Odesa y las regiones de Lugansk y Donetsk tuvieron lugar fuertes protestas, debido también a la imposición de nuevos gobernadores, oligarcas ucranianos que colaboraron en el golpe de Estado dado a Yanukovich y que fueron impuestos por el primer ministro interino Arseni Yatseniuk inmediatamente después del golpe.

Por eso, la población mayoritariamente rusa de Crimea después de proclamar los poderes internos su independencia de Ucrania, votaron por reintegrarse a Rusia y no padecer la política racista y violenta de los nacionalistas.

La perspectiva de uno de los más reconocidos analistas de geopolítica en Rusia, Alexander Duguin, plantea que Rusia debe crear el espacio económico euroasiático con las repúblicas exsoviéticas y una de las más importantes es Ucrania (Duguin, 2007). 
Alexander Duguin plantea que los procesos de integración económica euroasiática no han podido desarrollarse debido a que:

Rusia debe, por supuesto, responder desarrollando un nueva política hacia los Estados Unidos, que están detrás del aislamiento de Rusia en el espacio postsoviético, propiciando una integración inmediata de las repúblicas que todavía pueden integrarse y dejar de lado todas las demás consideraciones. Pero lo más importante ni siquiera es esto, porque en muchos sentidos la razón de todos los fracasos es la presencia de una quinta columna, una columna pro-estadounidense en Rusia misma, que sabotea cualquier proceso económico y procesos políticos positivos en nuestro país, interrumpe todos los aspectos positivos y los proyectos e iniciativas constructivas del presidente. Este es un grupo de pro estadounidenses, agentes de influencia en la persona de analistas y ultraliberales que no permiten plantear la cuestión de la necesidad de una verdadera modernización del sector real de la economía rusa, lo que haría mucho más fácil los procesos de integración (Duguin, 2007, p. 48).

Precisamente a estos ultraliberales pertenecía el político Boris Nemtsov, muy ligado a los intereses estadounidenses en Rusia, que fue asesinado, no se sabe con claridad por qué causa, y dejó como legado un informe (Informe Nemtsóv, 2015).

La UE también aplicó sanciones financieras y económicas a Rusia por la reintegración de Crimea y presionó a Bulgaria para que no autorizara el tránsito para un nuevo gasoducto denominado South Stream que abastecería a Europa central y oriental (Ukraine, 1994).

Entonces la estrategia de Rusia cambió y al mejorar la relación con Turquía, durante una visita de Vladimir Putin a Turquía en 2015, el gobierno ruso canceló el proyecto South Stream y acordó con el presidente Erdogan construir un gasoducto a través del lecho marino del Mar Negro hasta la frontera con Turquía bajo el proyecto de la compañía "Gazprom Rusa”. Este proyecto se denomina Turquish Stream. 
Hay que anotar que Turquía compra una cantidad significativa de gas ruso a través del territorio ucraniano, pero el sistema de transportación de Ucrania ha ido quedando obsoleto y, después de 2019, no podrá seguir en funcionamiento. Una de las causas de esta situación es que después del golpe de Estado, al presidente Víctor Yanukovich en 2014, el país no tiene voluntad política ni recursos para la reparación de este ducto.

En estas circunstancias, el gasoducto turco podría ser una buena alternativa para abastecer gas natural para Europa central y sur, pero la UE prácticamente ha puesto trabas a esta opción. El proyecto siguió adelante y el $1^{\circ}$ de diciembre de 2014, la sociedad pública rusa de acciones Gazprom y la compañía gubernamental Botas firmaron un memorándum para la construcción de un gasoducto con capacidad anual de 63 mil millones de metros cúbicos de gas.

En enero de 2015 tuvo lugar un encuentro del director de $G a-$ zprom Alexander Miller y el vicepresidente de la Comisión Europea para la unión energética, M. Shefchovich. Durante el curso de esta reunión, Miller declaró que el gasoducto Turquish Stream sería la única posibilidad para Europa de garantizar el abastecimiento de gas, evadiendo el territorio ucraniano. A su vez, el representante de la Comisión Europea informó que ni Turquía ni los países de Europa central y del este tenían intenciones de elevar la adquisición de gas y la construcción de un nuevo gasoducto sería objeto de un detallado análisis de las necesidades de los países europeos de este gas. Una vez más se observa cómo los intereses geopolíticos intervienen en los desarrollos de nuevas rutas del gas en Europa.

En Europa, el anuncio de este nuevo proyecto se interpretó como el intento de Rusia de seguir conservando el monopolio de abastecimiento del gas a este continente. Aunque el nuevo ducto proponía la construcción, según las nuevas normas europeas, de que la infraestructura será construida por los países donde el ducto pretende transitar. 


\section{El gasoducto Fuerza Siberia}

Debido a todos estos eventos, hay que anotar también que el eurocentrismo ya no es el único vector geográfico del complejo de petróleo y gas de Rusia. Así, el desarrollo del vector asiático de gasoductos le permite a Rusia tener nuevos mercados y así ampliar significativamente el margen de maniobra en las negociaciones con Europa sobre el precio del gas. Además, la unión de las provincias productoras de Siberia occidental y oriental permitirá transitar la exportación del oeste al este, a Asia, dependiendo de la coyuntura mundial.

El agudo empeoramiento de la situación geopolítica debido a la larga crisis por el conflicto de Ucrania y a la reabsorción de Crimea por Rusia, como se ha mencionado, llevó a un distanciamiento de las relaciones de Rusia con Europa. Esto influyó en la parte de la élite rusa más proclive ideológicamente a Occidente, que se rehusaba a exportar gas a Asia y que está presente en la compañía Gazprom que extrae el gas de Siberia occidental. Esta élite empezó a acercarse a la óptica de los siloviki (funcionarios de los servicios militares, de inteligencia y de seguridad) de repensar a donde dirigir la política energética exterior.

Para esta óptica actual, el tercer paquete energético de la UE representa un riesgo para las perspectivas de exportación del gas ruso a Europa y, por lo tanto, Rusia está ahora "girando" hacia Asia, lo que, en esencia, significa un giro hacia China, cuyo ascenso está cambiando el equilibrio general en la región y más allá. Existe un gran interés para Rusia en la realización de la iniciativa del Cinturón Económico de la Ruta de la Seda, ya que esta iniciativa es un factor para expandir la propia economía de Rusia y un factor para desarrollar la Unión Económica Eurasiática.

Rusia, por otra parte, está evidentemente centrada en las oportunidades políticas y económicas inherentes a los cambios tectónicos en esta parte del mundo. Originalmente atraída simplemente por la dinámica evolución económica en Asia y China, pero después de la crisis de Ucrania y las sanciones occidentales, su interés en el Oriente se ha convertido en una necesidad. Hoy en día, el pivote ruso de Asia y China se ha convertido en una 
parte integral de un importante cambio de paradigma con implicaciones más amplias.

Por otra parte, también el tercer paquete energético representa un riesgo para las perspectivas de exportación del gas ruso a Europa, ya que los gasoductos alemanes OPAL y NEL que son la continuación de la vía marítima del Nord Stream a tierra, no pueden llevar toda su capacidad de transporte de gas, ya que el Tercer paquete energético europeo no permite utilizar toda su capacidad, a pesar de que fueron contratados antes de que esta normativa entrara en vigor ( 55 mil millones de metros cúbicos al año). Todos los intentos de Moscú de lograr la autorización de la Comisión Europea para aumentar el volumen de abastecimiento de gas ruso por el Nord Stream no han sido positivas, aunque Moscú aduce que esta sería la alternativa al gasoducto que va de la isla Yamal y atraviesa Ucrania. Así, cada vez más la UE busca abastecimientos alternativos a Rusia, por lo que el gasoducto Fuerza de Siberia es una alternativa para que Rusia siga teniendo ingresos e influencia.

Inicialmente, Rusia esperaba construir un gasoducto desde Altai hasta el oeste de China, lo que le permitiría realizar entregas a China desde los mismos depósitos en Siberia occidental, desde donde se suministra gas a Europa occidental. Rusia podría entonces desempeñar el papel de un "productor compensador" y adquirir la máxima carta de triunfo política y económica en la regulación de los flujos de gas entre Europa y Asia. Rusia también negociaba con China el precio del gas que pagan los europeos, que es más alto que lo que esta paga a Turkmenistán.

Sin embargo, China insistió en que se instalara el gasoducto siberiano en el noreste de China, ya que tiene un gasoducto que va a la provincia de Xinjiang, en el oeste de la provincia de China, desde Turkmenistán. Además, experimenta una escasez de gas en sus provincias orientales densamente pobladas e industrializadas.

Para asegurar el suministro de gas, China quiere tener su propia fuente exclusiva de gas dedicada en el este de Siberia, que no sea compartida con Europa. Además, las compañías energéticas chinas necesitan ser compensadas por sus pérdidas en el mercado interno (donde los controles de precios en recursos energéti- 
cos son muy estrictos y los precios son menores a los del mercado mundial) al invertir en exploración, desarrollo y operación. Por lo tanto, China buscó el precio más bajo posible para minimizar las pérdidas internas y lo más probable es que Rusia recibe menos dinero por la venta de gas a China que a Europa.

Gazprom tiene la intención de convertirse en el mayor proveedor de gas para China, cubriendo más del $25 \%$ de sus necesidades para el año 2035, a medida que crezca la demanda de gas natural del país. China es el mayor importador de petróleo del mundo y el segundo mayor comprador de gas natural (Sputnik, 2019).

Según el politólogo ruso Dmitri Lekuj, "Europa perderá la capacidad de chantajear a Rusia con ser el "único mercado significativo", mientras que Rusia, con su gas relativamente barato en comparación con el gas natural licuado, va a convertirse en un socio mucho más importante. Un incentivo adicional para superar la posición negociadora anticuada con Europa será también el hecho de que China se ha convertido en el mayor importador de gas natural en el mundo por primera vez en el 2018.

El volumen de las importaciones de gas de China ascendió a 125.700 millones de metros cúbicos, lo que supone un $31 \%$ (30.300 millones de metros cúbicos) más que en el 2017. Además, esta importación no hará más que aumentar. Incluso el disputado proyecto de Nord Stream 2 podría haberse cancelado si Rusia no hubiera mostrado la estrategia de girar hacia Oriente, incluyendo su enfoque en Sila Sibiri, opina el analista” (Latuj, 2019).

\section{Reflexiones finales}

A pesar de los esfuerzos de Estados Unidos y la UE por bloquear el desarrollo de las nuevas rutas energéticas de Rusia, el gobierno de Vladimir Putin ha logrado desarrollar este importante componente de su economía que le ha permitido junto con el desarrollo de un nuevo poderío armamentista reposicionarse como una gran potencia mundial.

El objetivo fundamental de Occidente es, por lo tanto, trazar oleoductos y gasoductos en donde Rusia no participe, ni que atraviesen su territorio, como el BTC (Bakú, Tbilisi, Ceyhán) y el 
anterior proyecto Nabucco, que por ahora se ha abandonado. En un principio, Irán intentó participar en Nabucco, pero las acusaciones occidentales sobre su actividad de enriquecimiento de uranio impidieron llevar a cabo su participación. Irán posee $16 \%$ de las reservas mundiales de gas, solo después de Rusia, pero para Occidente, la candidatura de Irán está categóricamente excluida.

Así, la UE, considerando la dificultad para allegarse el gas del Caspio, buscaba alimentar el proyecto Nabucco con gas del Medio Oriente. Bruselas firmó el acuerdo sobre energía con el gobierno de Irak sobre el abastecimiento de gas iraquí como una entrega complementaria para Nabucco.

A esto contribuyó un activo lobby estadounidense y aunque este abastecimiento solo representaba la mitad del volumen requerido, también se traducía en grandes complicaciones geopolíticas para Occidente, ya que el ducto hubiera tenido que atravesar la región kurda y esto no lo aprobaría Ankara. Además, Irán tiene y tendrá una fuerte influencia en Irak que ampliará la minoría chiíta iraquí, y esto no lo desean ni Turquía, ni Estados Unidos.

Así, a casi treinta años de existencia de la Rusia postsoviética, mostraron la ilusión de la esperanza por parte de Occidente de convertirla en un socio menor de Estados Unidos y de la UE. Sin fundamento resultaron también los esfuerzos llevados a cabo por Washington y por los mismos liberales rusos después de la desintegración de la URSS, de que no volviera a ser uno de los principales actores de la política mundial, por lo que, "la propia tradición geopolítica de Rusia, su sistema de relaciones exteriores, sus características territoriales, militares, de poderío armamentista y culturales de gran potencia, que no pueden ser revisados y cambiados a voluntad, no pueden ser alterados trátese de quien se trate que lo intente" (Duguin, 2007).

Por lo tanto, ninguna intención subjetiva ha tenido la capacidad de mover al país del denominado heartland de Mackinder. Su existencia en el espacio continental euroasiático como una especificidad inseparable del destino de Rusia, imprime su huella en la elección de sus habitantes, de su forma de vida y de los caminos de desarrollo del país (Duguin, 2007). 


\section{Referencias}

Agencia Rusa de Noticias. (2019, 12 de mayo). El gasoducto turco estará en explotación a finales de 2019. Турецкий поток введут в эксплуат Русское Агентство Новостей. httр:// xn----ctbsbazhbctieai.ru-an.info

Ansley, R. (2017, 03 de mayo). Making the Three Seas Initiative a Priority for Trump. Atlantic Council. https://www.atlanticcouncil.org/blogs/new-atlanticist/making-the-three-seas-initiative-a-priority-for-trump

Carbajosa, A. (2017, 16 de junio). Alemania acusa a EEUU de querer beneficiar a sus empresas con las sanciones a Rusia. El País: https://elpais.com/internacional/2017/06/15/actualidad /1497548830_862699.html

Carmona, I. G. (2019, 07 de junio). Las implicaciones de la construcción de Nord Stream 2 para la Unión de la Energía de la Unión Europea. Centro Español de Estudios Estratégicos. http://www.ieee.es/Galerias/fichero/docs_opinion/2019/ DIEEEO52_2019ISAGAC-NordStream2.pdf

Comité Estatal de Estadísticas, GOSKOMSTAT. (1991). Economía Nacional e Industria. Gobierno de Rusia.

Deutsche Welle. (2018, 03 de agosto). El director de Naftogaz: las sanciones de Estados Unidos pueden detener el Nord Stream 2. Deutsche Welle. https://www.dw.com/ru/\%D0\%B4\%D0\%B8\%D1\%80\%D0\%B5\%D0\%BA\%D1\%82\%D0\%BE\%D1\%80-\%D0\%BD\%D0\%B0\%D1\%84\%D1\%82\%D0\%BE\%D0\%B3\%D0\%B0\%D0\%B7\%D0\%B0-D0\%BD\%D0\%BA\%D1\%86\%D0\%B8\%D0\%B8-\%D1\%81\%D1\%88\%D0\%B0-\%D0\%BC\%D0\%BE\%D0\%B3\%D1\%83\%D1\%82-\%D0\%BE\%D1\%81\%D1\%82\%D0\%B0\%D0\%BD\%D0\%BE

Duguin, A. (2007). Геополитика Постмодерна (Geopolítica Postmoderna). Ed. Amfora.

Evseev, V. (2015). Turquish Stream: Genesis and Prospects . Continente Postsoviético, 43-54.

Financial Times. (2006, 29 de octubre). US criticises Russia Germany Gas Deal. Financial Times. https://www.ft.com/ content/50e6faec-6779-11db-8ea5-0000779e2340 
Gobierno de Rusia. (2005). Estrategia Energética de Rusia hasta 2020. https://docs.cntd.ru/document/901872984

Index Mundi Country Facts. (2017). Natural Gas probed reserves, hwww.indexmundi.com/factbook/fields/natural-gas-proved-reserves Index Mundi Country Facts. (2017). Reservas probadas de petróleoporpais, https://www.indexmundi.com/map/?v=97\&l=es Khasanov. (2017). Draft of the bill called "On energy diplomacy and security." United States Senate, 2007.

Intelectualnaia Rossia. (2007, 27 de marzo). Revisión de la Politica Exterior de Rusia, http://www.intelros.ru/strategy/gos_ rf/316-obzor_vneshnejj_politiki__podgotovlennyjj_ministerstvom_inostrannykh_del_rossii.html

Latuj,D. (2019, 17 defebrero). Rusiasuministragas a China antes delo previsto: ¿qué significa esto para Europa? Sputnik Mundo, https:// mundo.sputniknews.com/economia/201902171085537191-rusia-china-fuerza-siberia-gas-europa/

Maskevich, I. (2018, 11 de marzo). En cinco países firmaron una carta sobre el peligro de Nord Stream 2. ZN,UA. https:// zn.ua/ECONOMICS/v-pyati-stranah-evropy-podpisali-pismo-ob-opasnosti-severnogo-potoka-2-277671_.html

Milosevich-Juaristi, M. (2016, 15 de julio). El proceso de 'reimperialización' de Rusia 2000-2016, Real Instituto Elcano DT 11/2016. http://www.realinstitutoelcano.org/wps/portal/rielcano_es/ contenido?WCM_GLOBAL_CONTEXT=/elcano/elcano_es/ zonas_es/dt11-2016-milosevichjuaristi-proceso-reimperializacion-rusia-2000-2016

Milosevich-Juaristi, M. (2019, 09 de mayo). Los aliados de Rusia: su ejército, su armada y su gas. Real Instituto Elcano ARI 47/2019. http://www.realinstitutoelcano.org/wps/portal/rielcano_es/contenido?WCM_GLOBAL_CONTEXT=/elcano/elcano_es/zonas_es/ari47-2019-milosevichjuaristi-aliados-de-rusia-su-ejercito-su-armada-y-su-gas

Palazuelos, E. (1993). Problemas Energéticos en la antigua Unión Soviética : La economía rusa en 1993. En Consecuencias del primer año de reformas, Informe elaborado por el Observatorio Económico Permanente (pp. 109-178). Instituto de Europa Oriental. 
Pusenkova, H. (2012). La industria petrolera rusa: 20 años que conmovieron al mundo. Economia Mundial y Relaciones Internacionales, 2072.

Informe Nemtsóv (2015, 20 de agosto). Putin es la guerra. El informe Nemtsóv sobre el conflicto de Ucrania. Frontera $D$ Revista Digital. https://www.fronterad.com/putin-es-la-guerra-el-informe-nemtsov-sobre-el-conflicto-de-ucrania/

Rar, A. (2011, 15 de octubre). El gasoducto del norte: la gran política de un proyecto comercial. BFM.RU. https://www.bfm.ru/ news/ 157153

RT. (2016, 25 de agosto). Russia's Nord Stream-2 pipeline is a 'bad deal' for Europe-Biden. RT. https://www.rt.com/business/357176-nord-stream2-biden-us-russia/

Sergeiev, P. (1998). Transporte a través de gasoductos y oleoductos de Rusia y Europa Occidental. Economia Mundial y Relaciones Internacionales, 11, 113.

Singotiya, P. (2018, 02 de noviembre). What is CAATSA? Quora. https://www.business-standard.com/article/pti-stories/lawmakers-discussing-about-impact-of-caatsa-on-allies-us-senator-118052200108_1.html

Telguian, E. (1999). La industria petrolera de Rusia en condiciones de disminución de los precios en el mercado mundial del petróleo. Economía Mundial y Relaciones Internacionales, Academia de Ciencias de Rusia.

Tsujlo, S. (2005). Situación Económica y Política en Rusia en 2007. Instituto de la Economía en Transición, Moscú, Rusia, 4-17.

Ukraine, R. F. (1994, 05 de diciembre). Memorandum on security assurances in connection with Ukraine's accession to the Treaty.treaties. un.org/doc/Publication/UNTS: https:/treaties.un.org/doc/Publication/ UNTS/No\%20Volume/52241/Part/I-52241-0800000280401fbb.pdf US Department of Treasury. (2017, 02 de agosto). Countering America's Adversaries Through Sanctions Act. US Department of Treasury. https://www.treasury.gov/resource-center/ sanctions/Programs/Pages/caatsa.aspx 